\title{
Diagnostic accuracy of diagnostic imaging for lumbar disc herniation in adults with low back pain or sciatica is unknown; a systematic review
}

Jung-Ha Kim,2, Rogier M. van Rijn ${ }^{1,3}$, Maurits W. van Tulder ${ }^{4,5}$, Bart W. Koes ${ }^{1}$, Michiel R. de Boer ${ }^{4}$, Abida Z. Ginai ${ }^{6}$, Raymond W. G. J. Ostelo ${ }^{4,5}$, Danielle A. M. W. van der Windt ${ }^{7}$ and Arianne P. Verhagen ${ }^{3,8^{*}}$

\begin{abstract}
Main text: We aim to summarize the available evidence on the diagnostic accuracy of imaging (index test) compared to surgery (reference test) for identifying lumbar disc herniation (LDH) in adult patients.

For this systematic review we searched MEDLINE, EMBASE and CINAHL (June 2017) for studies that assessed the diagnostic accuracy of imaging for LDH in adult patients with low back pain and surgery as the reference standard. Two review authors independently selected studies, extracted data and assessed risk of bias. We calculated summary estimates of sensitivity and specificity using bivariate analysis, generated linked ROC plots in case of direct comparison of diagnostic imaging tests and assessed the quality of evidence using the GRADE-approach.

We found 14 studies, all but one done before 1995, including 940 patients. Nine studies investigated Computed Tomography $(C T)$, eight myelography and six Magnetic Resonance Imaging (MRI). The prior probability of LDH varied from 48.6 to $98.7 \%$. The summary estimates for MRI and myelography were comparable with CT (sensitivity: $81.3 \%$ (95\%Cl $72.3-87.7 \%)$ and specificity: $77.1 \%(95 \% \mathrm{Cl} 61.9-87.5 \%))$. The quality of evidence was moderate to very low.

Conclusions: The diagnostic accuracy of $C T$, myelography and MRI of today is unknown, as we found no studies evaluating today's more advanced imaging techniques. Concerning the older techniques we found moderate diagnostic accuracy for all CT, myelography and MRI, indicating a large proportion of false positives and negatives.
\end{abstract}

Keywords: Diagnostic accuracy, Systematic review, Lumbar disc herniation, Diagnostic imaging, Low back pain

\section{Main text}

\section{Introduction}

Approximately $5-15 \%$ of patients with low back pain suffer from lumbar disc herniation (LDH) $[1,2]$. LDH is the most common spine disorder requiring surgical intervention [3, 4]. Clinical guidelines recommend history taking and physical examination to rule out LDH diagnosis [4]. However, the diagnostic accuracy of both history taking and physical examination is still insufficient $[5,6]$. Diagnostic imaging in patients with back

\footnotetext{
* Correspondence: Arianne.verhagen@uts.edu.au

${ }^{3}$ Department of Public Health, Erasmus University Medical Center Rotterdam, Rotterdam, The Netherlands

${ }^{8}$ School of Physiotherapy, Graduate school of Health, University Technology Sydney, Sydney, Australia

Full list of author information is available at the end of the article
}

pain and/or leg pain is often used to assess nerve root compression due to disc herniation or spinal stenosis and cauda equina syndrome [7-10]. Furthermore, diagnostic imaging can also be used to identify the affected disc level before surgery [11].

Diagnostic imaging can be done by Magnetic Resonance Imaging (MRI), Computed Tomography (CT), $\mathrm{X}$-ray and myelography. Currently MRI is the imaging modality of choice, as it has the advantage of not using ionising radiation and has good visualizing capacities especially of soft tissue $[9,12]$. CT is often used and available for detection of morphologic changes and has a well-recognized role in the diagnosis of herniated discs $[13,14]$. Compared to MRI, CT is cheaper, the total testing time is shorter, and the availability of CT scanners is

(c) The Author(s). 2018 Open Access This article is distributed under the terms of the Creative Commons Attribution 4.0 International License (http://creativecommons.org/licenses/by/4.0/), which permits unrestricted use, distribution, and 
larger in hospital settings, but has the drawback of exposure to ionising radiation. Myelography involves injection of contrast medium in the lumbar spine, followed by X-ray, CT or MRI projections [15]. For certain conditions (e.g. metal implants or malalignment of the spine) myelography might replace MRI as the imaging modality of choice [16]. Plain radiography (X-ray) is the most commonly used technique due to its relative low cost and ready availability $[9,17-19]$.

However, the evidence for diagnostic accuracy of diagnostic imaging for LDH is still unclear [20, 21]. In addition, discordance between patients' clinical findings and MRI findings is also reported [22, 23]. We have performed a large study evaluating the evidence om diagnostic accuracy of MRI and CT for all kinds of lumbar pathologies compared to various reference standards $[12,24]$. The aim of the current review is to more specifically summarize and compare the evidence on the diagnostic accuracy of diagnostic imaging (CT, X-rays, myelography and MRI) identifying LDH in patients with low back pain and/or leg pain with surgery as a reference standard.

\section{Methods}

\section{Design}

A systematic review and meta-analysis, according to the guidelines of the Cochrane handbook of systematic reviews of diagnostic test accuracy studies [25]. The protocol was registered in PROSPERO (2015:CRD42015027687).

\section{Search strategy}

We conducted the search in MEDLINE, EMBASE, and CINAHL (untill 1 June 2017) without language restriction (see Appendix 1). The search strategy was designed in collaboration with a medical information specialist. In addition, reference lists of relevant review articles as well as all retrieved relevant publications on diagnostic test accuracy studies were checked to identify any potentially missed articles.

\section{Study selection}

We applied the following selection criteria: a) both prospective and retrospective cohort and case-control studies; b) adults with low back and/or leg pain with lumbar disc herniation as the suspected underlying pathology; c) Index tests were MRI, X-ray, myelography or CT; d) Reference standard was surgery; e) Data to generate $2 \times 2$ table; f) Published full reports, preferably in English, Dutch or German language.

We defined LDH as herniated nucleus pulposus, including protruded, extruded or sequestrated disc, causing nerve root compression. Two of the review authors (RvR/RO/BK/JHK/MB) independently selected first titles and abstracts and assessed relevant full papers. We used consensus to resolve disagreements; in cases of persisting disagreement a third review author (AV) was consulted.

\section{Risk of bias assessment}

Pairs of review authors (MvT/BK/RvR/JHK) independently performed risk of bias assessment using the Quality Assessment of Diagnostic Accuracy Studies (QUADAS)-2 tool [26]. In the flow and timing domain, we considered a time period between index test and reference standard of 1 week or less appropriate. Risk of bias and concerns about applicability of each domain were classified as low, high or unclear risk. Consensus was reached by discussion of discrepancies between the two reviewers. If discrepancies persisted, we consulted a third reviewer (AV).

\section{Data extraction}

Pairs of review authors (MvT/BK/JHK/RvR) independently performed data extraction using a standardised form. We extracted data on author, year of publication and journal; study design and setting; study population; pathology considered, age, gender, numbers of subjects for inclusion in study and analysis, patient selection, level of measurement (patient or disc). Also, we obtained data on index and reference test characteristics; including type of test, year; methods of execution, cut- off values, positivity thresholds and outcome scales; diagnostic parameters; diagnostic two-by-two table or parameters to reconstruct this table.

\section{Statistical analysis}

For each included study we calculated sensitivity and specificity (and 95\% confidence intervals (CI)) preferably on patient level data using the data from two-by-two tables. We conducted a meta-analysis separately for each of the index tests using a bivariate analysis. We chose the bivariate random-effects approach, because it incorporates both within and between study variation of sensitivity and specificity together with any correlation that might exist between sensitivity and specificity [27]. We present summary point estimates of sensitivity and specificity (and 95\% confidence region) and the results were plotted in receiver operating characteristic (ROC) space [28]. When possible we generated linked ROC plots in case of pairs of diagnostic imaging tests, when both tests had been evaluated in the same study. Meta-regression was used to evaluate whether there is a difference in test accuracy between different imaging techniques or between patient level data and disc level data [29]. Analysis was carried out using STATA 13.1 software.

Two reviewers (JHK, AV) assessed the quality of the evidence for each index test using the Grading of Recommendations Assessment, Development, and Evaluation (GRADE) working group criteria [28, 30]. Disagreements were resolved by a third review author $(\mathrm{MB} / \mathrm{DvdW})$. The 
quality of evidence is categorized as high, moderate, low, or very low [31]. The quality of the evidence started at high and is reduced by one level for each of the following domains not met: limitations of the study design ( $>25 \%$ of participants in studies with two or more domains with high risk of bias); indirectness ( $>25 \%$ of participants in studies with serious applicability concerns); inconsistency (unexplained variation in sensitivities and specificities across the studies [32]); imprecision (wide confidence interval of the sensitivity and specificity in $>25 \%$ of the studies); and publication bias [33].

\section{Results}

Literature search

A total of 27,776 citations were obtained. Finally, 14 studies met our selection criteria (Fig. 1). No studies were excluded based on the language. Of these, nine studies investigated CT [34-42], eight myelography [34, 37-39, 41, 43], six MRI [36, 39, 43-46], and none assessed X-ray. All studies were performed in secondary care settings, such as neurological clinics or pain clinics; three studies [41, 43, 47] were retrospective (Table 1). All but one study evaluated old imaging

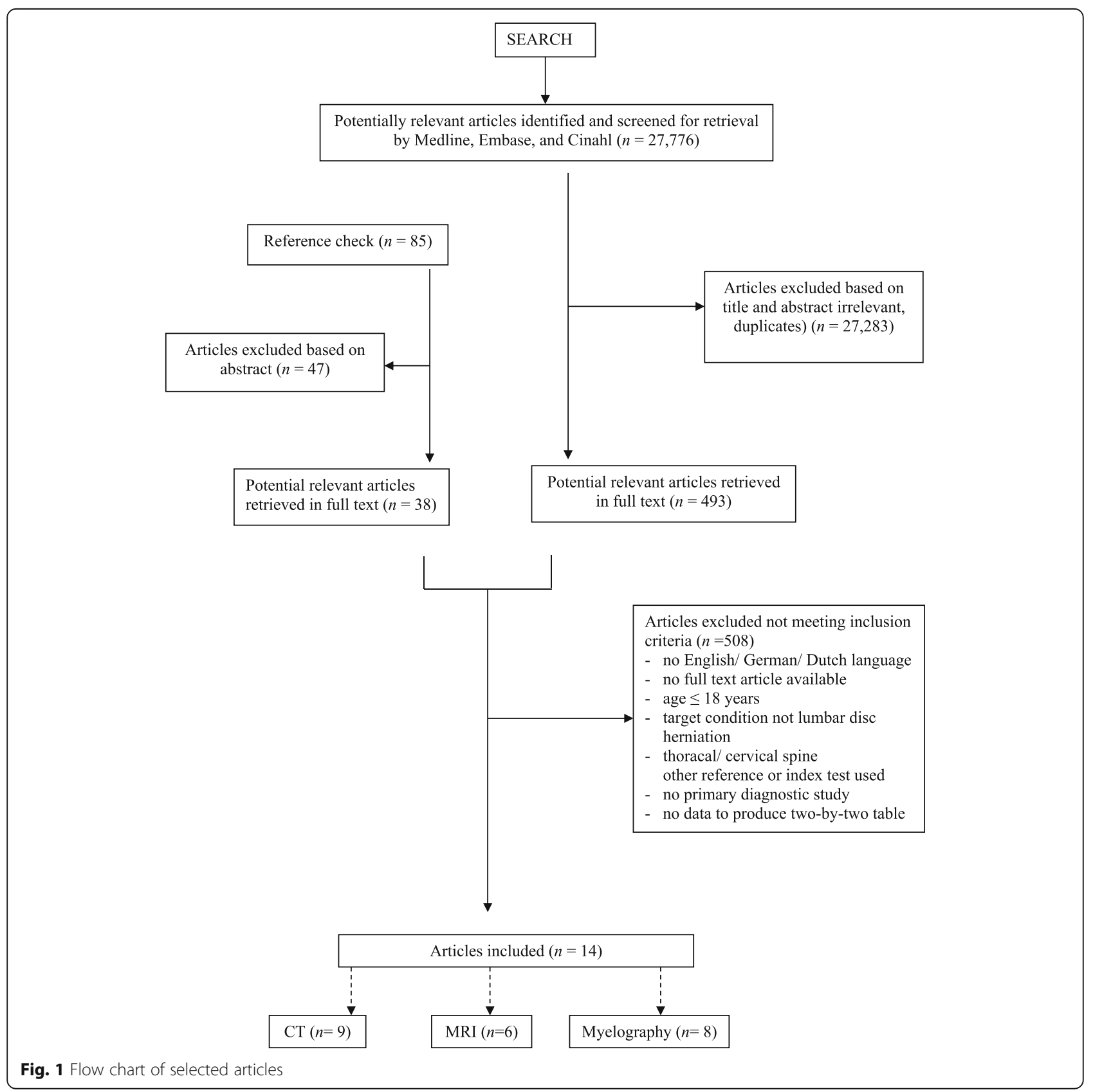




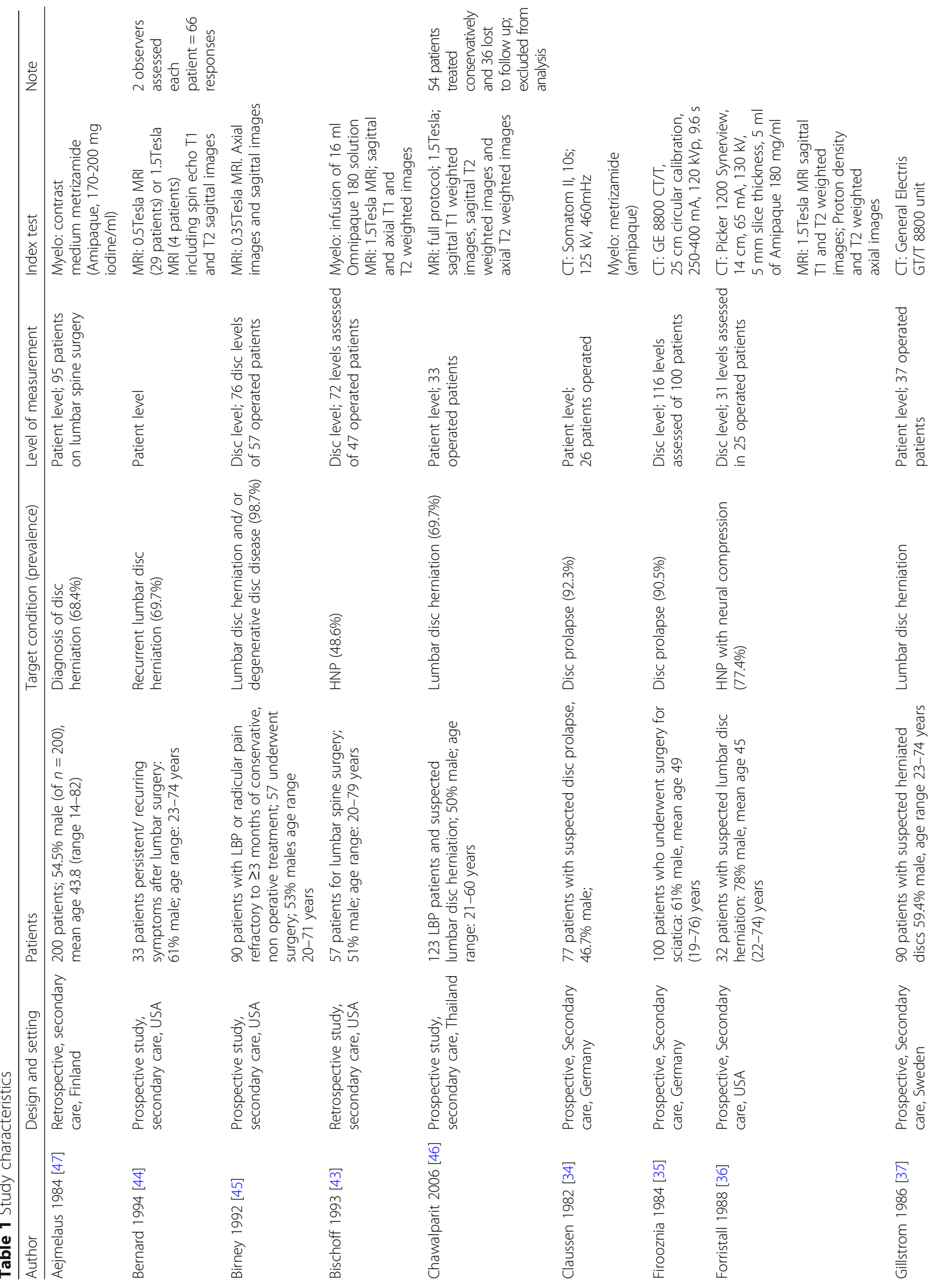




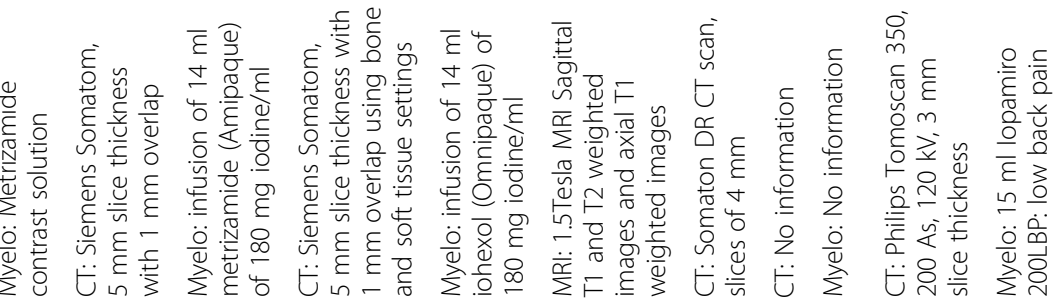

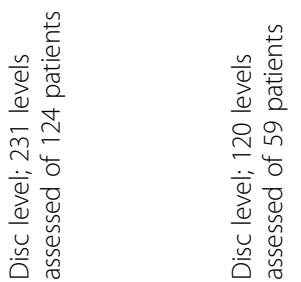

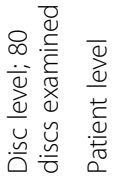

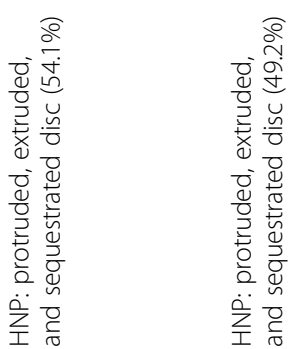

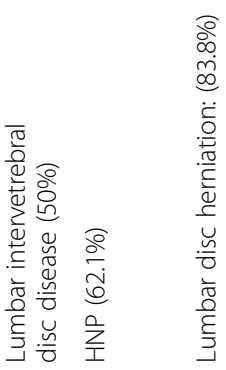

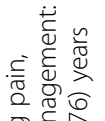

政

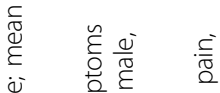

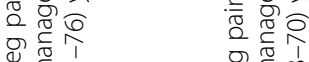

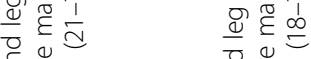

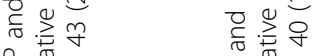

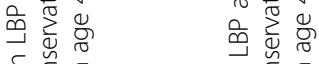

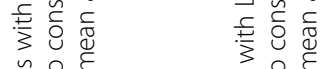

है욜

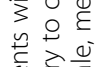

岕产高

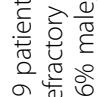

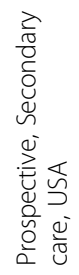

in 0 i 늉

बiv

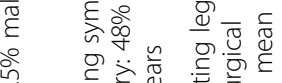

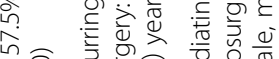

ஸे

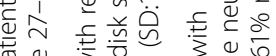

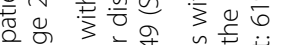

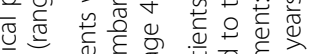

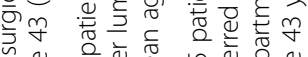

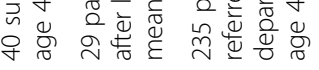
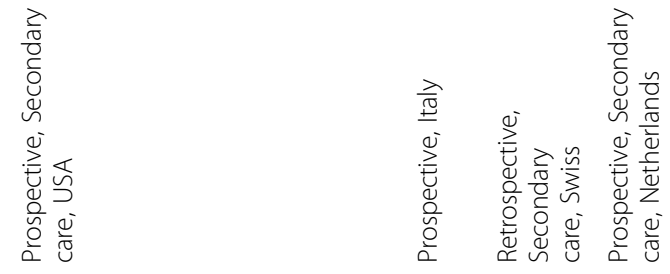

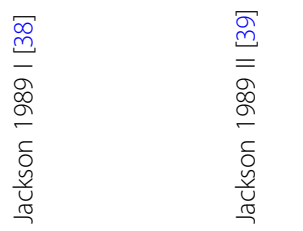

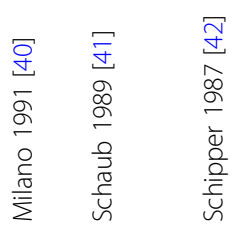


techniques as they were published between 1982 and 1994, one study evaluating MRI was published in 2006 [46].

\section{Population}

A total of 940 patients receiving surgery were included. Overall 1289 patients were involved in these studies but the reference standard was not performed in 349 patients. The patients (14 to 82 years) all had clinical findings consistent with LDH. Seven studies $(n=288)$ $[34,37,41,42,44,46,47]$ were analyzed on patient level; others analyzed disc levels (Table 1 ).

\section{Risk of bias}

Although we only selected studies using surgery as a reference standard, none of the studies were assessed as having low risk of bias $(\mathrm{RoB})$ related to the reference standard, mainly because it was unclear whether results of the reference standard had been interpreted without knowledge of imaging results (Fig. 2). Seven studies were considered to have high RoB related to patient selection, as patients had not clearly been selected using consecutive or random sampling. Only two studies reported a time-interval between index test and reference standard, which were 3 months and 9 months, respectively [44, 47].

\section{Diagnostic accuracy \\ Computed tomography}

Nine studies, with four studies with measurements on patient level (327 patients) [34, 37, 41, 42] and a total of 578 discs explorations $[35,36,38-40]$, were included. The mean prior probability of LDH was $72.0 \%$ (range 49.2-92.3\%). The sensitivity and specificity ranged from 59 to $93 \%$ and from 45 to $100 \%$, respectively (Fig. 3). The summary estimates were $81.3 \%$ (95\%CI: $72.3-$ $87.7 \%$ ) for sensitivity and $77.1 \%$ (95\%CI: $61.9-87.5 \%)$ for specificity (Fig. 4). We found no inconsistency as an

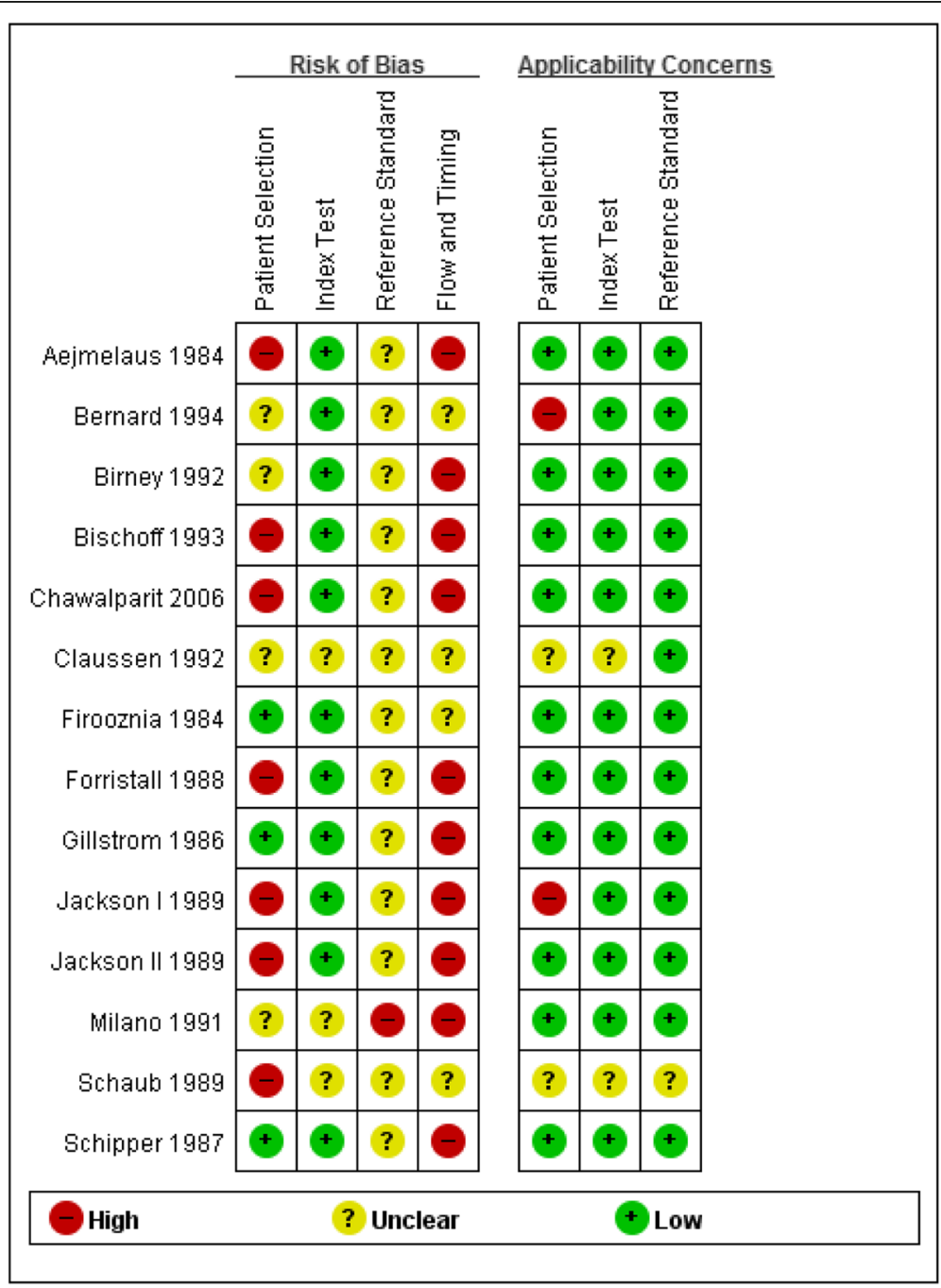

Fig. 2 Assessment of risk of bias for each included study 


\begin{tabular}{|c|c|c|c|c|c|c|c|c|}
\hline Study & TP & FP & FN & TN & Sensitivity $(95 \% \mathrm{Cl})$ & Specificity $(95 \% \mathrm{Cl})$ & Sensitivity $(95 \% \mathrm{Cl})$ & Specificity (95\% Cl) \\
\hline Claussen 1992 & 21 & 1 & 3 & 1 & $0.88[0.68,0.97]$ & $0.50[0.01,0.99]$ & & \\
\hline Firooznia 1984 & 97 & 4 & 8 & 7 & $0.92[0.86,0.97]$ & $0.64[0.31,0.89]$ & & \\
\hline Forristall 1988 & 20 & 2 & 4 & 5 & $0.83[0.63,0.95]$ & $0.71[0.29,0.96]$ & & \\
\hline Gillstrom 1986 & 28 & 3 & 2 & 4 & $0.93[0.78,0.99]$ & $0.57[0.18,0.90]$ & & \\
\hline Jackson I 1989 & 89 & 25 & 36 & 81 & $0.71[0.62,0.79]$ & $0.76[0.67,0.84]$ & & \\
\hline Jackson II 1989 & 35 & 8 & 24 & 53 & $0.59[0.46,0.72]$ & $0.87[0.76,0.94]$ & & \\
\hline Milano 1991 & 40 & 0 & 6 & 34 & $0.87[0.74,0.95]$ & $1.00[0.90,1.00]$ & & $\rightarrow$ \\
\hline Schaub 1989 & 13 & 6 & 5 & 5 & $0.72[0.47,0.90]$ & $0.45[0.17,0.77]$ & & \\
\hline Schipper 1987 & 140 & 5 & 57 & 31 & $0.71[0.64,0.77]$ & $0.86[0.71,0.95]$ & & \\
\hline
\end{tabular}

Fig. 3 Forest plot of the diagnostic accuracy of $\mathrm{CT}$ in the identification of lumbar disc herniation

inverse correlation between logit-transformed sensitivity and logit-transformed specificity was shown (estimate $=$ $-0.2649)$. There were no differences in summary estimates for sensitivity and/or for specificity between patient level data and disc level data (chi-square $=2.52$, 2df, $P=0.28)$.

We found a moderate quality evidence (downgraded because of limitations in study design) for the accuracy of CT (Table 2).

\section{Myelography}

Eight studies, with five studies with measurements on patient level (422 patients) [34, 37, 41, 42, 47] and a total 423 disc explorations [38, 39, 43], were included. The mean prior probability of LDH was $69.2 \%$ (range: 49.2-91.3\%). The sensitivity and specificity ranged from 54 to $92 \%$ and from 50 to $89 \%$, respectively (Fig. 5). We found a summary estimate of $75.7 \%$ (95\%CI: $64.9-84.1 \%)$ for sensitivity and $76.5 \%$ (95\%CI: 67.8-83.4\%) for specificity (Fig. 4). We found no inconsistency (estimate $=-0.7644$ ). There was a difference in summary estimate for sensitivity between patient level data (83.9\% (95\%CI: 76.4-89.3\%)) and disc level data (61.1\% (95\%CI: 50.2-71.0\%)) (chi-square $=9.23,1 \mathrm{df}, P=0.002)$, but not for specificity (chi-square $=1.26$, $1 \mathrm{df}, P=0.26$ ).

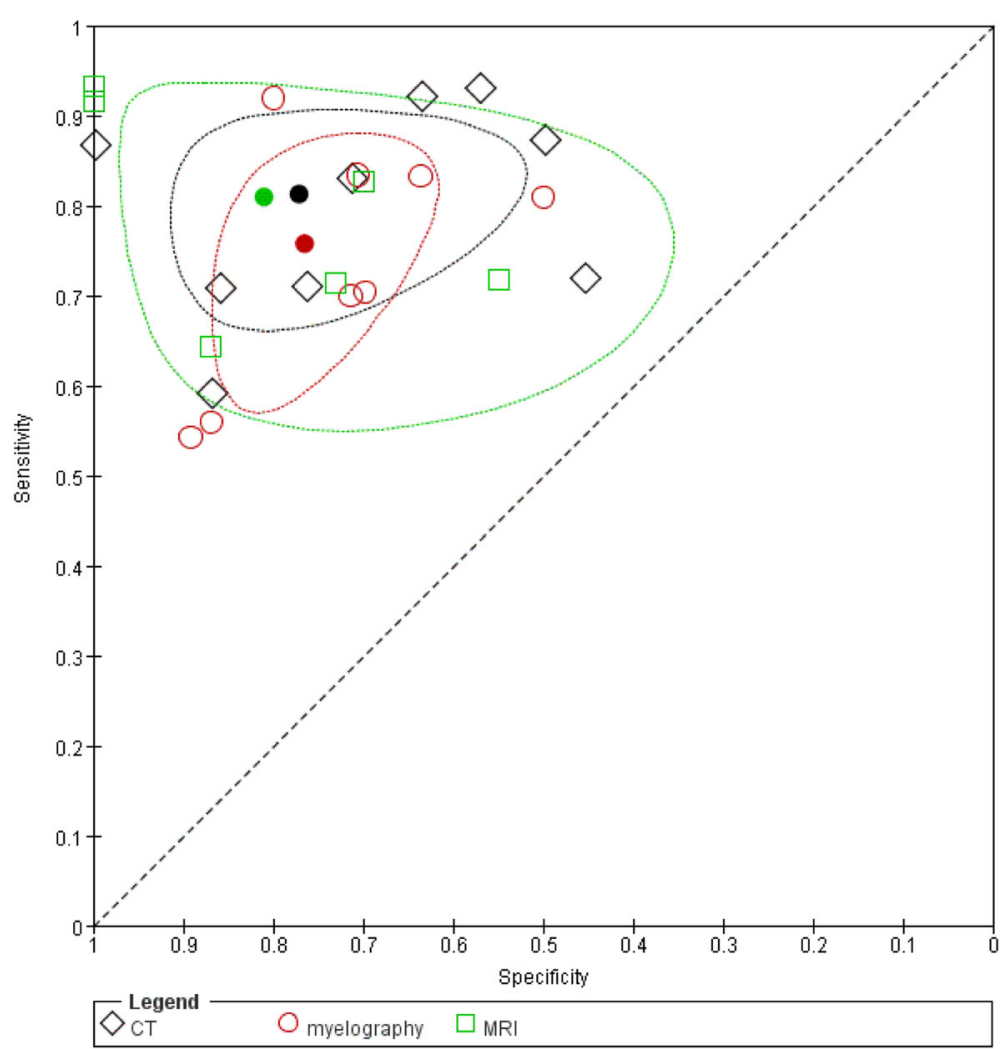

Fig. 4 Summary ROC plots of sensitivity and specificity of all studies 
Table 2 GRADE evidence for diagnostic accuracy of lumbar disc herniation

\begin{tabular}{|c|c|c|c|c|c|c|}
\hline & Study design & Indirectness & Inconsistency & Imprecision & Publication bias & Quality \\
\hline \multicolumn{7}{|l|}{$C T$} \\
\hline 9 studies & Serious limitation ${ }^{a}$ & $\mathrm{No}^{\mathrm{b}}$ & $\mathrm{No}^{\mathrm{c}}$ & $\mathrm{No}^{d}$ & $\mathrm{No}^{\mathrm{e}}$ & Moderate \\
\hline \multicolumn{7}{|c|}{ Myelography } \\
\hline 8 studies & Serious limitation ${ }^{a}$ & $\mathrm{No}^{\mathrm{b}}$ & $\mathrm{No}^{\mathrm{c}}$ & $\mathrm{No}^{d}$ & $\mathrm{No}^{\mathrm{e}}$ & Moderate \\
\hline \multicolumn{7}{|l|}{ MRI } \\
\hline 6 studies & Serious limitation ${ }^{a}$ & $\mathrm{No}^{\mathrm{b}}$ & Serious limitation ${ }^{c}$ & Serious limitation ${ }^{d}$ & $\mathrm{No}^{\mathrm{e}}$ & Very low \\
\hline
\end{tabular}

We conclude that there is moderate quality evidence for the accuracy of myelography (downgraded because of limitations in study design) (Table 2).

\section{Magnetic resonance imaging}

Six studies, with two studies with measurements on patient level (66 patients) [44, 46] and a total 299 disc explorations [36, 39, 43, 45], were included. In these studies the mean prior probability of LDH was $68.9 \%$ (range: 48.6-98.7\%). The sensitivity and specificity ranged from 64 to $93 \%$ and from 55 to $100 \%$, respectively with wide confidence intervals (imprecision) (Fig. 6). The summary estimate was $80.9 \%$ (95\%CI: $68.8-89.1 \%$ ) for sensitivity and $81 \%$ (95\%CI: $59.2-92.6 \%)$ for specificity (Fig. 4). Because of a positive correlation between logit-transformed sensitivity and logit-transformed specificity (estimate $=0.5516$ ) we decided that there was inconsistency. It was not possible to examine a difference between patient level data and disc level data in sensitivity and specificity.

We conclude that there is very low quality evidence for the accuracy of MRI (downgraded by study design, inconsistency and imprecision) (Table 2).

\section{Comparing imaging techniques CT versus Myelography}

Six studies evaluated CT and myelography (followed by plain radiography) in the same patient population and the linked results are plotted in ROC space (Fig. 7) [34, 37-39, 41, 42]. The summary estimate of sensitivity was $76.7 \%$ (95\%CI: $66-84.8 \%$ ) for CT and $74.4 \%$ (95\%CI: $64.8-82.2 \%$ ) for myelography. The summary estimate of specificity was $71.2 \%$ (95\%CI: $55.2-83.2 \%$ ) for CT and was $72.4 \%$ (95\%CI: 62.5-80.4\%) for myelography. These summary estimates were slightly lower compared to the ones based on all CT and myelography studies. We concluded that there is comparable accuracy for CT and myelography (chi square $=0.27$, 2df, $P=0.87)$.

\section{$C T$ versus $M R I$}

Two studies evaluated CT and MRI (Fig. 8) [36, 39]. The summary estimate of sensitivity was $70.6 \% \quad(95 \% \mathrm{CI}$ : 49.5-85.5\%) for CT and $80.0 \%$ (95\%CI: $50.6-93.9 \%$ ) for MRI. The summary estimate of specificity was $82.5 \%$ (95\%CI: 63.3-92.7\%) for CT and 93.5\% (95\%CI: $57.0-$ 99.4\%) for MRI. The results showed a comparable accuracy for CT and MRI (chi-square $=0.51,2 \mathrm{df}, P=0.78$ ).

\section{Myelography versus MRI}

Two studies evaluated myelography and MRI (Fig. 9) $[39,43]$. The summary estimate of sensitivity was $55.3 \%$ (95\%CI: 45.2-65.0\%) for myelography and $67.4 \%$ (95\%CI: 56.6-76.7\%) for MRI. The summary estimate of specificity was $87.8 \%$ (95\%CI: 79.7-92.9\%) for myelography and $81.3 \%$ (95\%CI: $69.4-89.3 \%)$ for MRI. These

\begin{tabular}{|c|c|c|c|c|c|c|c|c|}
\hline Study & TP & FP & FN & TN & Sensitivity (95\% Cl) & Specificity (95\% Cl) & Sensitivity (95\% Cl) & Specificity (95\% Cl) \\
\hline Aejmelaus 1984 & 91 & 5 & 8 & 20 & $0.92[0.85,0.96]$ & $0.80[0.59,0.93]$ & $\rightarrow$ & \\
\hline Bischoff 1993 & 19 & 4 & 16 & 33 & $0.54[0.37,0.71]$ & $0.89[0.75,0.97]$ & & \\
\hline Claussen 1992 & 17 & 1 & 4 & 1 & $0.81[0.58,0.95]$ & $0.50[0.01,0.99]$ & & \\
\hline Gillstrom 1986 & 21 & 2 & 9 & 5 & $0.70[0.51,0.85]$ & $0.71[0.29,0.96]$ & & \\
\hline Jackson I 1989 & 88 & 32 & 37 & 74 & $0.70[0.62,0.78]$ & $0.70[0.60,0.78]$ & & \\
\hline Jackson II 1989 & 33 & 8 & 26 & 53 & $0.56[0.42,0.69]$ & $0.87[0.76,0.94]$ & & \\
\hline Schaub 1989 & 15 & 4 & 3 & 7 & $0.83[0.59,0.96]$ & $0.64[0.31,0.89]$ & & \\
\hline Schipper 1987 & 191 & 10 & 38 & 24 & $0.83[0.78,0.88]$ & $0.71[0.53,0.85]$ & \begin{tabular}{cccccc}
1 & 1 & & \multicolumn{1}{|l}{} \\
0 & 0.2 & 0.4 & 0.6 & 0.8 & 1
\end{tabular} & \begin{tabular}{ccccc} 
& & & \multicolumn{1}{c}{} \\
0 & 0.2 & 0.4 & 0.6 & 0.8
\end{tabular} \\
\hline
\end{tabular}

Fig. 5 Forest plot of the diagnostic accuracy of myelography 


\begin{tabular}{|c|c|c|c|c|c|c|c|c|}
\hline Study & TP & FP & FN & TN & Sensitivity (95\% Cl) & Specificity ( $95 \%$ Cl) & Sensitivity ( $95 \% \mathrm{Cl})$ & Specificity $(95 \% \mathrm{Cl})$ \\
\hline Bernard 1994 & 33 & 9 & 13 & 11 & $0.72[0.57,0.84]$ & $0.55[0.32,0.77]$ & & \\
\hline Birney 1992 & 70 & 0 & 5 & 1 & $0.93[0.85,0.98]$ & $1.00[0.03,1.00]$ & & \\
\hline Bischoff 1993 & 25 & 10 & 10 & 27 & $0.71[0.54,0.85]$ & $0.73[0.56,0.86]$ & & -1 \\
\hline Chawalparit 2006 & 19 & 3 & 4 & 7 & $0.83[0.61,0.95]$ & $0.70[0.35,0.93]$ & & \\
\hline Forristall 1988 & 22 & 0 & 2 & 7 & $0.92[0.73,0.99]$ & $1.00[0.59,1.00]$ & & \\
\hline Jackson II 1989 & 38 & 8 & 21 & 53 & $0.64[0.51,0.76]$ & $0.87[0.76,0.94]$ & & 1 \\
\hline
\end{tabular}

Fig. 6 Forest plot of the diagnostic accuracy of MRI

results indicate comparable accuracy for myelography and MRI (chi-square $=3.59,2 \mathrm{df}, P=0.17$ ).

\section{Discussion}

We found 14 diagnostic accuracy studies including 940 patients and all evaluating rather old imaging techniques. Summary estimates of sensitivity and specificity of the different imaging techniques varied between 76 and $81 \%$, with moderate to very low quality evidence. Furthermore, CT, myelography and MRI show comparable accuracy.

We found very low quality evidence for diagnostic accuracy of MRI. Even though MRI is more expensive, clinicians generally prefer MRI to CT, as it does not carry the risks associated with ionising radiation and unlike myelography, MRI is non-invasive [48]. MRI may also be more useful when surgical treatment is considered as it can identify tissue properties as well as anatomical structures [48]. These are most likely the reasons for suggesting MRI as the most appropriate test to confirm the presence of LDH in a recent guideline regardless its disappointing diagnostic accuracy.

\section{Strengths and weaknesses}

Heterogeneity arises from several reasons. First, imaging techniques used in studies included old ones like 0.5 Tesla [44] or 0.35 Tesla MRI [45]. In clinical practice the results of diagnostic imaging are interpreted with knowledge of history items and physical examination. Furthermore, clinicians frequently state that imaging does not play a crucial role in predicting prognosis or deciding on a management strategy among patients with

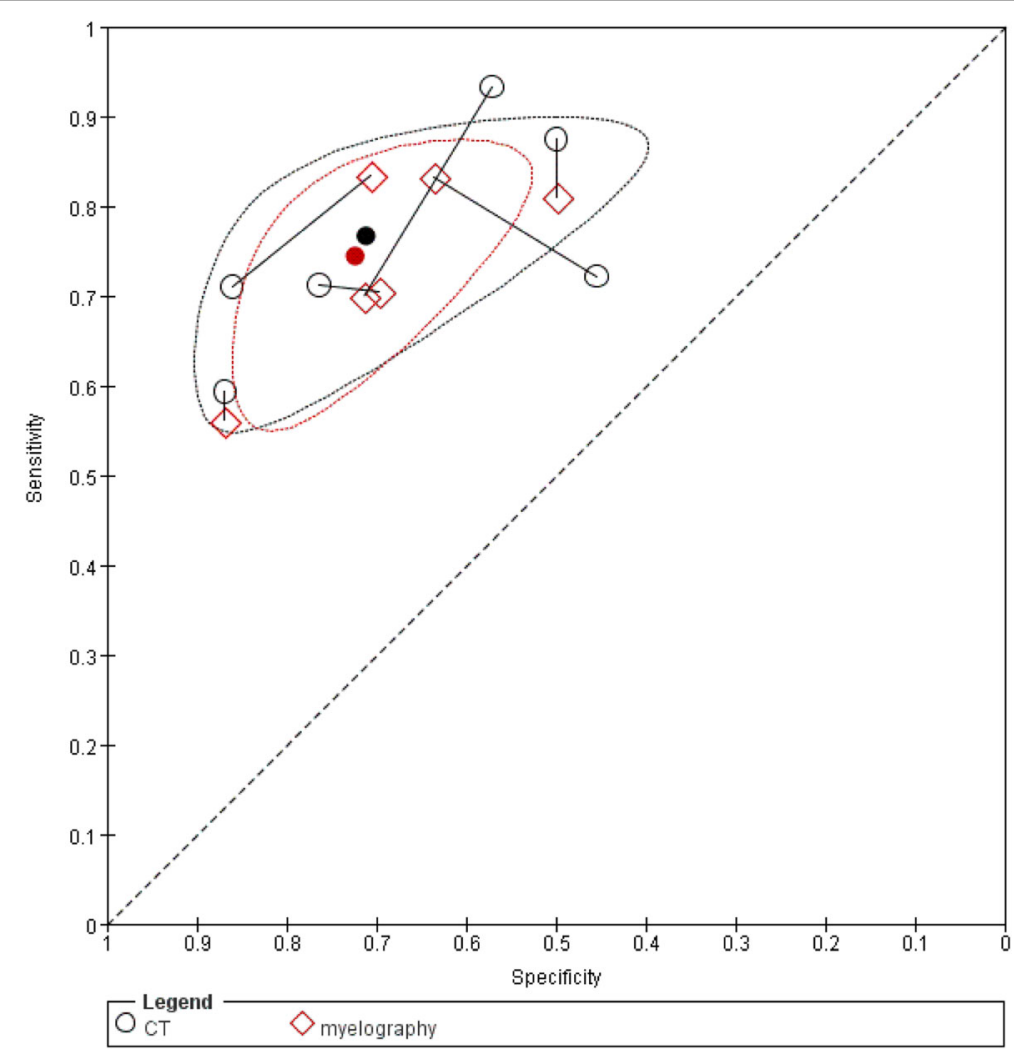

Fig. 7 Summary ROC plots of $C T$ versus myelography 


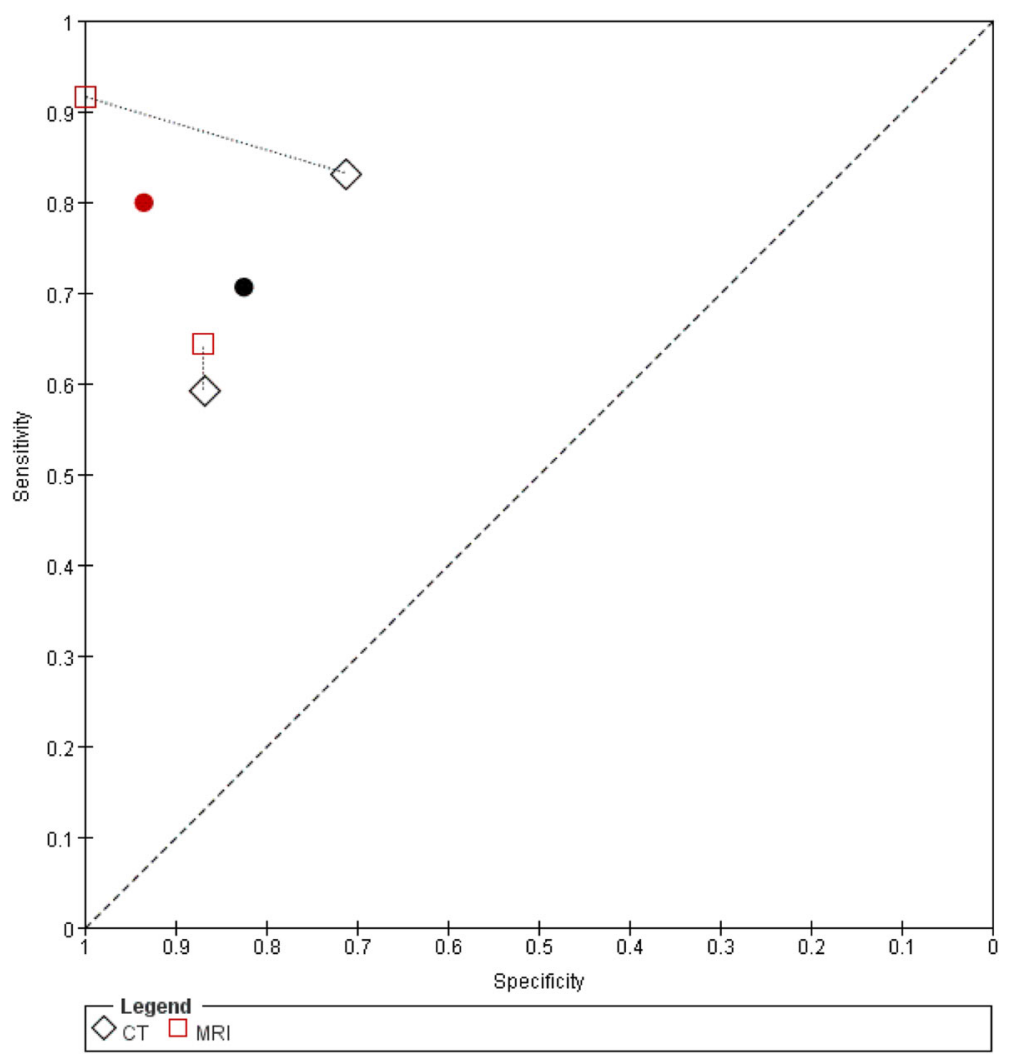

Fig. 8 Summary ROC plots of $C T$ versus MRI

LDH [4]. This might be one of the reasons why there are no recent studies on the diagnostic accuracy of imaging techniques for detecting LDH. However, older techniques will probably identify less underlying causes of back pain than newer imaging techniques. Evaluation of diagnostic accuracy of advanced diagnostic equipment is therefore needed. Second, the included studies focussed on LDH, but classification of this pathology differed between studies [49]. For example, some studies defined LDH as protruded, extruded, and sequestrates disc $[38,39]$, but other studies were defined LHD as the presence of neuronal compression [35, 36, 42, 46]. There were some studies without a definition of LHD [37, 40]. Third, we combined disc level data with patient level data. Results at disc level including more than one disc level in the same patient may lead to smaller confidence intervals and possibly to an overestimation of diagnostic accuracy. Unexpectedly, confidence intervals were often wider in disc level data compared to patient level data. Fourth, the diagnostic accuracy in this study was possibly overestimated by a high prior probability (48.6 to $98.5 \%)$ of LDH. It was reported that about $4 \%$ of patients who present with low back pain in a primary care setting have a disc herniation [8]. The high prior probability results in selection bias. Furthermore, patient selection was unclear in many studies. This is important since the interpretation of the test result (posterior probability) depends on its sensitivity and specificity as well as the probability of the disease [50]. Lastly, the use of surgery as a reference standard can easily bias the results due to partial verification [51]. Surgery is often regarded as the best available reference standard. Not everyone is subjected to surgery but only those patients with a very strong suspicion based on clinical symptoms combined with the results of the diagnostic imaging of LDH which leads to (partial) verification bias. In this review, among 669 patients with suspected LDH, 349 (52.2\%) patients did not undergo surgical treatment in seven studies [34, 36, 37, 43, 45-47]. Verification bias can lead to an increased diagnostic accuracy of the index test; i.e. it will show an increased sensitivity.

As far as we know, this is the first meta-analysis comparing diagnostic accuracy between different techniques in low back and/or leg pain with LDH as the suspected underlying pathology.

\section{Implications}

Concerning practice we conclude that the diagnostic accuracy of today's imaging techniques in unknown. This severely hampers the choice of techniques as well as the interpretation of the outcomes as no information is present concerning false positives or negatives. Future 


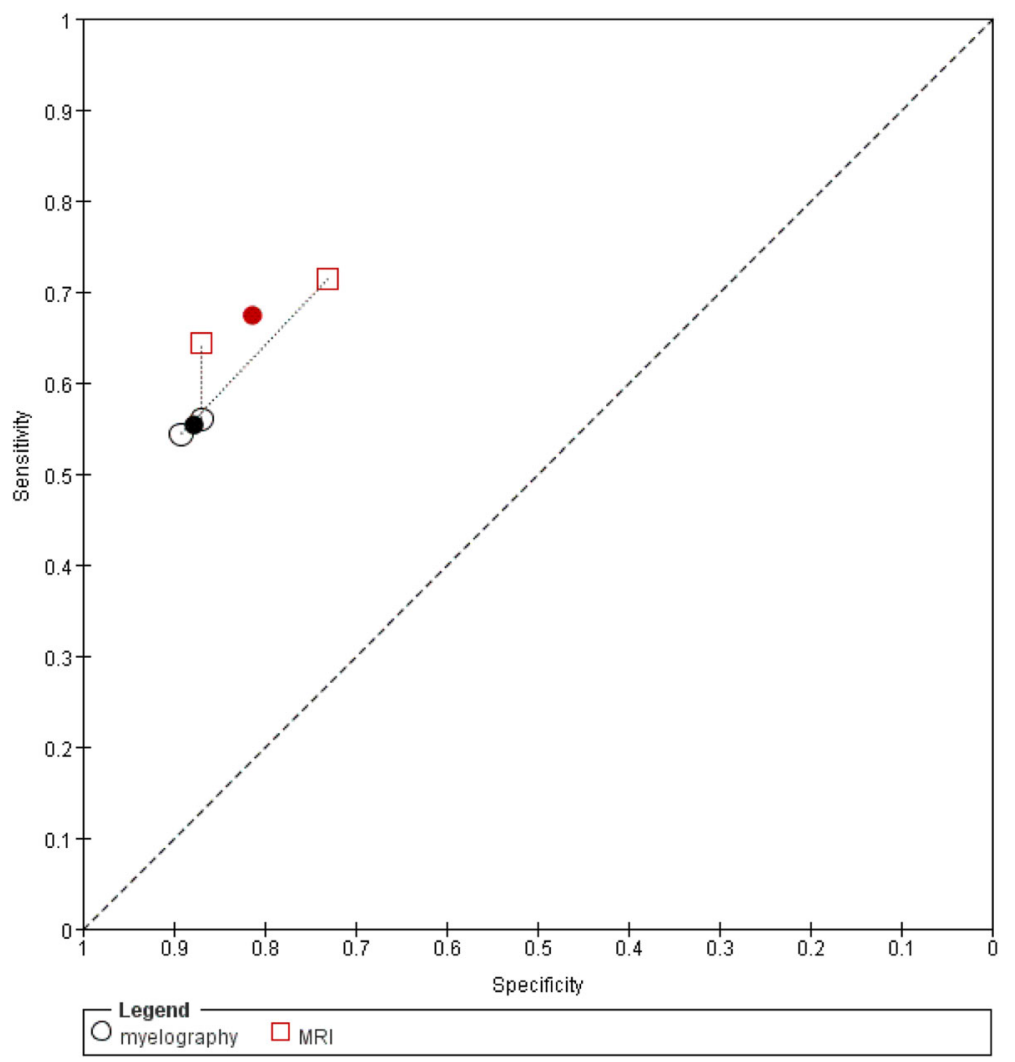

Fig. 9 Summary ROC plots of myelography versus MRI

research should focus on the diagnostic accuracy of frequently used imaging techniques (diagnostic test accuracy studies) and on the place of diagnostic imaging within the clinical pathway (diagnostic modelling).

\section{Conclusion}

In conclusion, we found no studies evaluating modern diagnostic imaging techniques. For the older techniques we found moderate quality evidence for moderate diagnostic accuracy of CT and myelography, and very low quality evidence for moderate diagnostic accuracy of MRI in patients with suspected lumbar disc herniation. The accuracy of CT, MRI and myelography is comparable.

\section{Appendix 1}

\section{Search strategy DTA imaging in low back pain Embase.com}

('nuclear magnetic resonance imaging'/exp OR 'computer assisted tomography'/exp OR radiography/exp OR 'diagnostic imaging'/exp OR radiodiagnosis/de OR ((magnet* NEAR/3 resonance ) OR mri OR nmri OR ((mr OR nmr) NEAR/3 imag*) OR (comput* NEAR/3 tomograph*) OR ct OR cat OR radiogra* OR (x NEXT/1 ray*) OR 'plain film' OR myelogra* OR (diagnos* NEAR/3 imag*) OR radiodiagnos $^{*}$ ):ab,ti) AND (backache/exp OR sciatica/exp
OR ('radicular pain'/exp AND (back/exp)) OR (((back OR

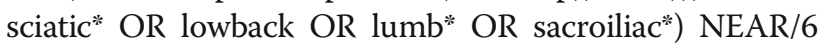
(ache OR pain* OR aching OR complaint* OR dysfunction* OR disabilit* OR trauma* OR symptom* OR injur* OR patholog* OR problem*)) OR (fail* NEAR/3 back NEAR/3 surg* ) OR backache* OR backpain* OR schiatica OR ischia* OR lumbago OR lumboischialgia OR ((radicular OR radiculalgi*) NEAR/6 (back OR spine* OR spinal*)) OR dorsalgi*):ab,ti) AND ('spine disease'/exp OR 'neurologic disease'/exp OR Osteoporosis/exp OR Osteoarthritis/ exp OR Osteosclerosis/exp OR (((spin* OR vertebra* OR intervertebr* OR disc* OR disk* OR neurologic* OR nerve* NEAR/3 (disease* OR injur* OR tumor* OR tumour* OR neoplas* OR cancer* OR malign* OR fracture* OR hernia* OR displace* OR protru* OR avuls* OR degenerat* OR Stenos* OR Osteophytos* OR entrap* OR compress* OR inflammat* OR disorder* OR rupture* OR disrupt*)) OR Radiculopath* OR polyradiculopath* OR Spondylarthrit* OR Spondyloarthrit* OR Spondylit* OR Spondylolisthes* OR Spondylolys* OR Discitis OR Osteoporo* OR Osteoarthrit* OR Osteosclero* OR Ankylos*):ab,ti) AND ('cohort analysis'/exp OR 'follow up'/exp OR 'longitudinal study'/ exp OR 'prospective study'/exp OR 'retrospective study'/ exp OR 'case control study'/exp OR 'cross-sectional study'/ de OR epidemiology/de OR (cohort* OR (follow* NEXT/1 
up*) OR followup* OR longitudinal* OR prospectiv* OR

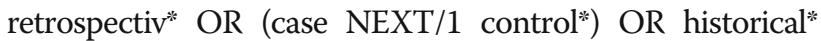
OR epidemiolog* OR (cross NEXT/1 section*)):ab,ti) NOT ((juvenile/exp NOT adult/exp)) NOT ([animals]/lim NOT [humans]/lim) NOT ([Conference Abstract]/lim OR [Letter]/lim OR [Note]/lim OR [Editorial]/lim OR 'systematic review'/exp OR 'case report'/exp OR ('systematic review' OR 'case report'):ti)

\section{Medline ovid}

(exp "magnetic resonance imaging"/ OR exp "Nuclear Magnetic Resonance"/ OR exp “Tomography, X-Ray Computed"/ OR exp radiography/ OR radiography.xs. OR "diagnostic imaging"/ OR radiodiagnosis/ OR ((magnet* ADJ3 resonance) OR mri OR nmri OR ((mr OR nmr) ADJ3 imag*) OR (comput* ADJ3 tomograph*) OR ct OR cat OR radiogra* OR (x ADJ ray*) OR "plain film" OR myelogra* OR (diagnos* ADJ3 imag*) OR radiodiagnos*).ab,ti.) AND (exp "back pain"/ OR sciatica/ OR ("Radiculopathy"/ AND (back/)) OR (((back OR sciatic" OR lowback OR lumb* OR sacroiliac*) ADJ6 (ache OR pain* OR aching OR complaint* OR dysfunction* OR disabilit* OR trauma* OR symptom* OR injur* OR patholog* OR problem*)) OR (fail* ADJ3 back ADJ3 surg* ) OR backache* OR backpain* OR schiatica OR ischia* OR lumbago OR lumboischialgia OR ((radicular OR radiculalgi*) ADJ6 (back OR spine* OR spinal*)) OR dorsalgi*).ab,ti.) AND (exp "Spinal Diseases"/ OR exp "Nervous System Diseases"/ OR Osteoporosis/ OR Osteoarthritis/ OR Osteosclerosis/ OR (((spin* OR verte-

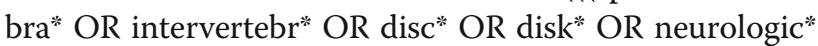
OR nerve ${ }^{*}$ ) ADJ3 (disease* OR injur* OR tumor* OR tumour* OR neoplas* OR cancer* OR malign* OR fracture $^{*}$ OR hernia* OR displace* OR protru* OR avuls* OR degenerat* OR Stenos* OR Osteophytos* OR entrap* OR compress* OR inflammat* OR disorder* OR rupture ${ }^{*}$ OR disrupt*)) OR Radiculopath* OR polyradiculopath* OR Spondylarthrit* OR Spondyloarthrit* OR Spondylit* OR Spondylolisthes* OR Spondylolys* OR Discitis OR Osteoporo* OR Osteoarthrit* OR Osteosclero* OR Ankylos").ab,ti.) AND ("Epidemiologic Studies"/ OR exp "Cohort Studies"/ OR "Case-Control Studies"/ OR "cross-sectional studies"/ OR (cohort* OR (follow* ADJ up*) OR followup* OR longitudinal* OR prospectiv* OR

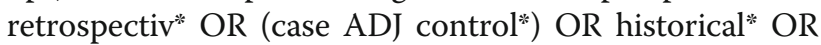
epidemiolog* OR (cross ADJ section*)).ab,ti.) NOT ((exp child/ NOT exp adult/)) NOT (exp animals/ NOT humans/) NOT ((Congresses OR Letter OR Notes OR Editorials).pt. OR "systematic review"/ OR "case report"/ OR ("systematic review" OR "case report").ti.)

\section{Web-of-science}

$\mathrm{TS}=\left(\left(\left(\left(\right.\right.\right.\right.$ magnet $^{*} \mathrm{NEAR} / 2$ resonance $)$ OR mri OR nmri OR ((mr OR nmr) NEAR/2 imag*) OR (comput* NEAR/ 2 tomograph $^{*}$ ) OR ct OR cat OR radiogra* OR (x NEAR/1 ray*) OR "plain film" OR myelogra* OR (diagnos* NEAR/2 imag*) OR radiodiagnos*)) AND (((back OR sciatic* OR lowback OR lumb* OR sacroiliac*) NEAR/5 (ache OR pain* OR aching OR complaint* OR dysfunction* OR disabilit* OR trauma* OR symptom* OR injur* OR patholog* OR problem*)) OR (fail* NEAR/2 back NEAR/2 surg* ) OR backache* OR backpain* OR schiatica OR ischia* OR lumbago OR lumboischialgia OR ((radicular OR radiculalgi*) NEAR/5 (back OR spine* OR spinal*)) OR dorsalgi*)) AND (((spin* OR vertebra* OR intervertebr* OR disc* OR

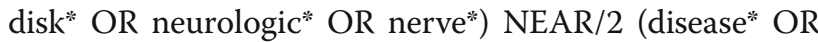
injur* OR tumor* OR tumour* OR neoplas* OR cancer* OR malign* OR fracture* OR hernia* OR displace* OR protru* OR avuls* OR degenerat* OR Stenos* OR Osteophytos* OR entrap* OR compress* OR inflammat* OR disorder* OR rupture* OR disrupt*)) OR Radiculopath* OR polyradiculopath* OR Spondylarthrit* OR Spondyloarthrit* OR Spondylit* OR Spondylolisthes* OR Spondylolys* OR Discitis OR Osteoporo* OR Osteoarthrit* OR Osteosclero* OR Ankylos*)) AND ((cohort* OR (follow* NEAR/1 (up OR ups)) OR followup* OR longitudinal* OR prospectiv* OR retrospectiv* OR (case NEAR/ 1 control*) $^{*}$ ) OR historical* OR epidemiolog* OR (cross NEAR/1 section*))) NOT ((child* OR infan* OR adoles-

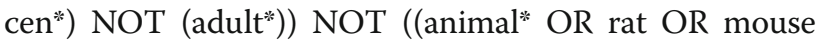
OR rats OR mice OR murine) NOT (human* OR patient*))) AND DT=(article) NOT $\mathrm{TI}=($ "systematic review" OR "case report")

\section{Pubmed publisher}

("magnetic resonance imaging" [mh] OR "Nuclear Magnetic Resonance"[mh] OR "Tomography, X-Ray Computed"[mh] OR radiography[mh] OR radiography[sh] OR "diagnostic imaging" [mh] OR radiodiagnosis[mh] OR (magnetic resonance*[tiab] OR mri OR nmri OR ((mr OR nmr) AND imag*[tiab]) OR (comput*[tiab] AND tomograph*[tiab]) OR ct OR cat OR radiogra*[tiab] OR x ray*[tiab] OR "plain film" OR myelogra*[tiab] OR (diagnos*[tiab] AND imag*[tiab]) OR radiodiagnos*[tiab])) AND ("back pain"[mh] OR sciatica[mh] OR ("Radiculopathy"[mh] AND (back[mh])) OR (((back OR sciatic*[tiab] OR lowback OR lumb*[tiab] OR sacroiliac*[tiab]) AND (ache OR pain*[tiab] OR aching OR complaint*[tiab] OR dysfunction*[tiab] OR disabilit*[tiab] OR trauma*[tiab] OR symptom*[tiab] OR injur*[tiab] OR patho$\log ^{*}[$ tiab] OR problem*[tiab])) OR (fail*[tiab] AND back AND surg*[tiab] ) OR backache*[tiab] OR backpain*[tiab] OR schiatica OR ischia*[tiab] OR lumbago OR lumboischialgia OR ((radicular OR radiculalgi*[tiab]) AND (back OR spine*[tiab] OR spinal*[tiab])) OR dorsalgi*[tiab])) AND ("Spinal Diseases"[mh] OR "Nervous System Diseases”[mh] OR Osteoporosis[mh] OR Osteoarthritis[mh] OR Osteosclerosis[mh] OR (() spine* $[$ tiab] OR spinal*[tiab] OR verteb$\mathrm{ra}^{*}[\mathrm{tiab}] \mathrm{OR}$ intervertebr*[tiab] OR disc[tiab] OR discs[tiab] OR disk*[tiab] OR neurologic*[tiab] OR nerve*[tiab]) AND 
(disease*[tiab] OR injur"[tiab] OR tumor*[tiab] OR tumour"[tiab] OR neoplas"[tiab] OR cancer"[tiab] OR malign*[tiab] OR fracture*[tiab] OR hernia*[tiab] OR displace*[tiab] OR protru*[tiab] OR avuls*[tiab] OR degenerat"[tiab] OR Stenos*[tiab] OR Osteophytos*[tiab] OR entrap*[tiab] OR compress*[tiab] OR inflammat*[tiab] OR disorder*[tiab] OR rupture*[tiab] OR disrupt*[tiab])) OR Radiculopath*[tiab] OR polyradiculopath*[tiab] OR Spondylarthrit*[tiab] OR Spondyloarthrit*[tiab] OR Spondylit*[tiab] OR Spondylolisthes*[tiab] OR Spondylolys"[tiab] OR Discitis OR Osteoporo*[tiab] OR Osteoarthrit*[tiab] OR Osteosclero*[tiab] OR Ankylos"[tiab])) AND ("Epidemiologic Studies"[mh] OR "Cohort Studies"[mh] OR "Case-Control Studies"[mh] OR "cross-sectional studies"[mh] OR (cohort"[tiab] OR follow up*[tiab] OR followup*[tiab] OR longitudinal"[tiab] OR prospectiv"[tiab] OR retrospectiv*[tiab] OR case control"[tiab] OR historical"[tiab] OR epidemiolog*[tiab] OR cross section*[tiab])) NOT ((child[mh] NOT adult[mh])) NOT (animals[mh] NOT humans[mh]) NOT (Congresses[pt] OR Letter[pt] OR Notes[pt] OR Editorials[pt] OR "systematic review" [mh] OR "case report"[mh] OR ("systematic review"[ti] OR "case report"[ti])) AND publisher[sb]

\section{Google scholar}

mri|ct|radiography|radiographically|"diagnostic imaging"|radiodiagnosis "back|lumbar pain"|backache cohort| "follow up"|longitudinal|prospective|retrospective|"case control"|epidemiological|"cross sectional"

\section{Acknowledgements}

We thank Merel Wassenaar for her useful help in designing the study and the data extraction and Wichor Bramer for developing the search strategy.

\section{Authors' contributions \\ $A V, M T, B K$ and $R O$ designed the study protocol, RvR, JHK, BK, RdB, RO and $A V$ were responsible for study selection, risk of bias assessment and data extraction. JHK, MdB, DvdW and AV were responsible for the analysis. AG acted as the content expert and all authors were responsible for reading and drafting the final version of the manuscript. All authors read and approved the final manuscript.}

\section{Ethics approval and consent to participate}

Not applicable.

\section{Consent for publication}

Not applicable.

\section{Competing interests}

The authors declare that they have no competing interest.

\section{Publisher's Note}

Springer Nature remains neutral with regard to jurisdictional claims in published maps and institutional affiliations.

\section{Author details}

'Department of General Practice, Erasmus University Medical Center, Rotterdam, The Netherlands. ²Department of Family Medicine, Chung-ang University Medical Center, 102, Heukseok-ro, Dongjak-gu, Seoul, South Korea. ${ }^{3}$ Department of Public Health, Erasmus University Medical Center Rotterdam, Rotterdam, The Netherlands. ${ }^{4}$ Department of Health Sciences and EMGO-Institute for Health and Care Research, Faculty of Earth \& Life Sciences, VU University Medical Centre, Amsterdam, The Netherlands. ${ }^{5}$ Department of Epidemiology and Biostatistics and EMGO-Institute for Health and Care
Research, VU University Medical Centre, Amsterdam, The Netherlands. ${ }^{6}$ Department of Radiology, Erasmus University Medical Centre, Rotterdam, Netherlands. ${ }^{7}$ Arthritis Research UK Primary Care Centre, Institute for Primary Care and Health Sciences, Keele University, Staffordshire, UK. ${ }^{8}$ School of Physiotherapy, Graduate school of Health, University Technology Sydney, Sydney, Australia.

Received: 19 April 2018 Accepted: 5 July 2018

Published online: 21 August 2018

\section{References}

1. Murray CJ, Barber RM, Foreman KJ, et al. Global, regional, and national disability-adjusted life years (DALYS) for 306 diseases and injuries and healthy life expectancy (HALE) for 188 countries, 1990-2013: quantifying the epidemiological transition. Lancet. 2015;386:2145-91.

2. Deyo RA, Loeser JD, Bigos SJ. Herniated lumbar intervertebral disk. Ann Intern Med. 1990;112:598-603.

3. Latka D, Miekisiak G, Jarmuzek P, Lachowski M, Kaczmarczyk J. Treatment of lumbar disc herniation with radiculopathy. Clinical practice guidelines endorsed by the polish Society of Spinal Surgery. Neurol Neurochir Pol. 2016;50:101-8.

4. Kreiner DS, Hwang SW, Easa JE, et al. An evidence-based clinical guideline for the diagnosis and treatment of lumbar disc herniation with radiculopathy. Spine J. 2014:14:180-91.

5. Verwoerd AJ, Peul WC, Willemsen SP, et al. Diagnostic accuracy of history taking to assess lumbosacral nerve root compression. Spine J. 2014;14:2028-37.

6. van der Windt DA, Simons E, Riphagen II, Ammendolia C, Verhagen AP, Laslett M, Devillé W, Deyo RA, Bouter LM, de vet HC, Aertgeerts B. Physical examination for lumbar radiculopathy due to disc herniation in patients with low-back pain: a systematic review. Cochrane Database Syst Rev. 2010; 2:CD007431

7. Lurie J, Tomkins-Lane C. Management of lumbar spinal stenosis. BMJ. 2016; 352:h6234.

8. Deyo RA, Rainville J, Kent DL. What can the history and physical examination tell us about low back pain? JAMA. 1992;268:760-5.

9. Jarvik JG, Deyo RA. Diagnostic evaluation of low back pain with emphasis on imaging. Ann Intern Med. 2002;137:586-97.

10. de Schepper El, Koes BW, Veldhuizen EF, Oei EH, Bierma-Zeinstra SM, Luijsterburg PA. Prevalence of spinal pathology in patients presenting for lumbar MRI as referred from general practice. Fam Pract. 2016;33:51-6.

11. Takashima $H$, Takebayashi $T$, Yoshimoto M, Terashima $Y$, Ida K, Yamashita T. Efficacy of diffusion-weighted magnetic resonance imaging in diagnosing spinal root disorders in lumbar disc herniation. Spine (Phila Pa 1976). 2013; 38:E998-E1002.

12. Wassenaar M, van Rijn RM, van Tulder MW, et al. Magnetic resonance imaging for diagnosing lumbar spinal pathology in adult patients with low back pain or sciatica: a diagnostic systematic review. Eur Spine J. 2012:21:220-7.

13. Hall FM. Back pain and the radiologist. Radiology. 1980;137:861-3.

14. Lurie JD. What diagnostic tests are useful for low back pain? Best Pract Res Clin Rheumatol. 2005;19:557-75.

15. Matsumoto T, Imagama S, Inoue H, Aoki T, Ishiguro N, Osawa Y. Outpatient Myelography: a prospective trial comparing complications after Myelography between outpatients and inpatients in Japan. Asian Spine J. 2015;9:928-34

16. Pomerantz SR. Myelography: modern technique and indications. Handb Clin Neurol. 2011;135:193-208.

17. Kendrick D, Fielding K, Bentley E, Kerslake R, Miller P, Pringle M. Radiography of the lumbar spine in primary care patients with low back pain: randomised controlled trial. BMJ. 2001;322:400-5.

18. Suthar P, Patel R, Mehta C, Patel N. MRI evaluation of lumbar disc degenerative disease. J Clin Diagn Res. 2015;9:TC04-9.

19. Jarvik JG, Hollingworth W, Martin B, et al. Rapid magnetic resonance imaging vs radiographs for patients with low back pain: a randomized controlled trial. JAMA. 2003:289:2810-8.

20. Deyo RA, Bigos SJ, Maravilla KR. Diagnostic imaging procedures for the lumbar spine. Ann Intern Med. 1989;111:865-7.

21. Ozturk C, Karadereler S, Ornek I, Enercan M, Ganiyusufoglu K, Hamzaoglu A. The role of routine magnetic resonance imaging in the preoperative evaluation of adolescent idiopathic scoliosis. Int Orthop. 2010;34:543-6. 
22. Chou R, Fu R, Carrino JA, Deyo RA. Imaging strategies for low-back pain: systematic review and meta-analysis. Lancet. 2009;373:463-72.

23. Mostofi K, Karimi Khouzani R. Reliability of the path of the sciatic nerve, congruence between Patients' history and medical imaging evidence of disc herniation and its role in surgical decision making. Asian Spine J. 2015; 9:200-4.

24. van Rijn RM, Wassenaar M, Verhagen AP, et al. Computed tomography for the diagnosis of lumbar spinal pathology in adult patients with low back pain or sciatica: a diagnostic systematic review. Eur Spine J. 2012;21:228-39.

25. Cochrane Handbook. Cochrane Handbook for Diagnostic Test Accuracy reviews. http://methods.cochrane.org/sdt/handbook-dta-reviews. Accessed 29 Nov 2016.

26. Whiting PF, Rutjes AW, Westwood ME. QUADAS-2: a revised tool for the quality assessment of diagnostic accuracy studies. Ann Intern Med. 2011; 155:529-36.

27. Reitsma JB, Glas AS, Rutjes AW, Scholten RJ, Bossuyt PM, Zwinderman AH. Bivariate analysis of sensitivity and specificity produces informative summary measures in diagnostic reviews. J Clin Epidemiol. 2005;58:982-90.

28. Guyatt $\mathrm{GH}$, Oxman AD, Vist $\mathrm{GE}$, et al. GRADE: an emerging consensus on rating quality of evidence and strength of recommendations. BMJ. 2008;336:924-6.

29. Takwoingi Y (2015) Meta-analysis using RevMan. http://imohw.tmu.edu.tw/ idohtmu/wp-content/uploads/2015/11/20151023_4-Meta-analysis-usingRevMan.pdf. Accessed 29 Nov 2016.

30. Balshem $H$, Helfand $M$, Schünemann $H J$, et al. GRADE guidelines: 3. Rating the quality of evidence. J Clin Epidemiol. 2011;64:401-6.

31. Atkins D, Best D, Briss PA, et al. Grading quality of evidence and strength of recommendations. BMJ. 2004;328:1490.

32. Harbord RM, Deeks JJ, Egger M, Whiting P, Sterne JA. A unification of models for meta-analysis of diagnostic accuracy studies. Biostatistics. 2007;8:239-51.

33. Schünemann HJ, Oxman AD, Brozek J, et al. Grading quality of evidence and strength of recommendations for diagnostic tests and strategies. BMJ. 2008;336:1106-10.

34. Claussen C, Grumme T, Treisch J, Lochner B, Kazner E. The diagnosis of lumbar disc prolapse. Computer tomographic and myelographic findings. Fortschr Geb Rontgenstr Nuklearmed. 1982;136:1-8.

35. Firooznia H, Benjamin V, Kricheff II, Rafii M, Golimbu C. CT of lumbar spine disk herniation: correlation with surgical findings. AJR Am J Roentgenol. 1984:142:587-92.

36. Forristall RM, Marsh HO, Pay NT. Magnetic resonance imaging and contrast $\mathrm{CT}$ of the lumbar spine. Comparison of diagnostic methods and correlation with surgical findings. Spine (Phila Pa 1976). 1988;13:1049-54.

37. Gillstrom P, Ericsson K, Hindmarsh T. A comparison of computed tomography and myelography in the diagnosis of lumbar disc herniation. Arch Orthop Traum Surg. 1986;106:12-4.

38. Jackson RP, Becker GJ, Jacobs RR, Montesano PX, Cooper BR, McManus GE. The neuroradiographic diagnosis of lumbar herniated nucleus pulposus: I. A comparison of computed tomography (CT), myelography, CT-myelography, discography, and CT-discography. Spine (Phila Pa 1976). 1989;14:1356-61.

39. Jackson RP, Cain JE Jr, Jacobs RR, Cooper BR, McManus GE. The neuroradiographic diagnosis of lumbar herniated nucleus pulposus: II. A comparison of computed tomography (CT), myelography, CT-myelography, and magnetic resonance imaging. Spine (Phila Pa 1976). 1989;14:1362-7.

40. Milano C, Lotti G, Mariconda M. Comparison of CT scan diagnosis and surgical findings in lumbar disk herniation. Ital J Orthop Traumatol. 1991;17:401-5.

41. Schaub B, Gratzl O, Bahous I. The value of myelography, computerized tomography and course of pain for the diagnosis of recurrent herniated disk. Schweiz Med Wochenschr. 1989;119:1185-90.

42. Schipper J, Kardaun JW, Braakman R, van Dongen KJ, Blaauw G. Lumbar disk herniation: diagnosis with CT or myelography. Radiology. 1987;165:227-31.

43. Bischoff RJ, Rodriguez RP, Gupta K, Righi A, Dalton JE, Whitecloud TS. A comparison of computed tomography-myelography, magnetic resonance imaging, and myelography in the diagnosis of herniated nucleus pulposus and spinal stenosis. J Spinal Disord. 1993;6:289-95.

44. Bernard TN Jr. Using computed tomography/discography and enhanced magnetic resonance imaging to distinguish between scar tissue and recurrent lumbar disc herniation. Spine (Phila Pa 1976). 1994;19:2826-32.

45. Birney TJ, White JJ Jr, Berens D, Kuhn G. Comparison of MRI and discography in the diagnosis of lumbar degenerative disc disease. J Spinal Disord. 1992;5:417-23.
46. Chawalparit O, Churojana A, Chiewvit P, Thanapipatsir S, Vamvanij V, Charnchaowanish P. The limited protocol MRI in diagnosis of lumbar disc herniation. J Med Assoc Thail. 2006;89:182-9.

47. Aejmelaeus R, Hiltunen $H$, Härkönen $M$, Silfverhuth $M$, Vähä-Tahlo T, Tunturi T. Myelographic versus clinical diagnostics in lumbar disc disease. Arch Orthop Traum Surg. 1984;103:18-25.

48. Taylor JA, Bussières A. Diagnostic imaging for spinal disorders in the elderly: a narrative review. Chiropr Man Therap. 2012;20:16.

49. Dionne CE, Dunn KM, Croft PR, et al. A consensus approach toward the standardization of back pain definitions for use in prevalence studies. Spine (Phila Pa 1976). 2008;33:95-103.

50. Erb HN. Prior probability (the pretest best guess) affects predictive values of diagnostic tests. Vet Clin Pathol. 2011;40:154-8.

51. Schmidt RL, Factor RE. Understanding sources of bias in diagnostic accuracy studies. Arch Pathol Lab Med. 2013;137:558-65.
Ready to submit your research? Choose BMC and benefit from:

- fast, convenient online submission

- thorough peer review by experienced researchers in your field

- rapid publication on acceptance

- support for research data, including large and complex data types

- gold Open Access which fosters wider collaboration and increased citations

- maximum visibility for your research: over $100 \mathrm{M}$ website views per year

At BMC, research is always in progress.

Learn more biomedcentral.com/submissions 